\title{
Vergiftungen in der Schweiz
}

\section{Stefan Weiler ${ }^{a}$, Hugo Kupferschmidt ${ }^{b}$}

${ }^{a}$ PD Dr. med., Leiter wissenschaftlicher Dienst, Tox Info Suisse; ${ }^{b}$ Dr. med., eMBA-HSG, Direktor Tox Info Suisse

\author{
Tox Info Suisse führte 2017 insgesamt 40310 Beratungen durch, gut 37200 Bera- \\ tungen zu Giftexpositionen und knapp 3100 prophylaktischer Natur.
}

54,5\% der Expositionen betrafen Kinder, mehrheitlich im Vorschulalter. Bei der Geschlechtsverteilung war bei den Kindern ein leichtes Überwiegen der Knaben (50,6\% vs. 48,1\% Mädchen) und bei den Erwachsenen der Frauen (58,1\% vs. 41,2\% Männer) zu sehen. 90\% der über 27000 unbeabsichtigten (akzidentellen) Vergiftungen ereigneten sich im häuslichen Milieu, bei den gut 4800 beabsichtigten Intoxikationen trat die grösste Anzahl (66\%) im Rahmen von Suizidversuchen auf.

\section{Schwere und tödliche Vergiftungen in der Schweiz 2017}

Bei den 246 schweren und tödlichen Vergiftungen überwiegt der Frauenanteil mit 57\%. Unterdurchschnittlich wenig Kinder sind betroffen (5\%). Die Patienten mit schweren Vergiftungen sind im Schnitt 40 Jahre alt, die mit tödlichem Ausgang 65 Jahre. Typischerweise ereignen sich die schweren und tödlichen Vergiftungen vorwiegend im Rahmen von beabsichtigten Handlungen (64\% suizidal, $13 \%$ Abusus). In über 85\% handelt es sich um eine orale Exposition.

\section{Medikamente}

Von 159 schweren [1] Vergiftungen mit Medikamenten (davon 6 Kinder) ereigneten sich 132 (83\%) mit Mitteln

\section{Das Wichtigste in Kürze}

- Im Jahr 2017 führte Tox Info Suisse 40310 Beratungen durch, rund 37200 zu Giftexpositionen und ca. 3100 waren prophylaktischer Natur.

- Über 18000 Giftexpositionen betrafen Kinder (vs. über 14000 bei Erwachsenen), 81\% davon jünger als 5 Jahre.

- Knapp drei Viertel (69\%) aller Vergiftungen geschahen mit Medikamenten, Haushaltsprodukten oder Pflanzen (Tab. 1).

- Vier (57\%) der sieben Todesfälle gingen auf das Konto der Medikamentenvergiftungen und je einer auf dasjenige von technischen/gewerblichen Produkten, Drogen sowie Nahrungsmittel/ Getränke (Tab. 2).

- Von den schweren Fällen waren 67\% durch Medikamente, 15\% durch Genussmittel und Drogen und 6\% durch Haushaltsprodukte verursacht. für das Nervensystem, im Wesentlichen Analgetika (v.a. Opioide, Paracetamol), Antiepileptika (am häufigsten Lamotrigin, Carpabamezin, Clonazepam und Phenobarbital) und Psychopharmaka (Benzodiazepine $\mathrm{n}=35$, Antidepressiva $\mathrm{n}=27$, Antipsychotika $\mathrm{n}=32$, davon Quetiapin $n=16$, Zolpidem $n=3$ ). Bei den übrigen schweren Medikamentenintoxikationen waren Präparate für den Gastrointestinaltrakt (Insulin $n=4$, Metformin bzw. Sitagliptin/Metformin je $n=1$ ), für den Kreislauf (Digoxin $n=2$ ), für den Atmungstrakt (Diphenhydramin $n=2$, Theophyllin $n=1$ ) und für den Bewegungsapparat (Mefenaminsäure $\mathrm{n}=2$, Baclofen $\mathrm{n}=2$, Tizanidin $\mathrm{n}=1$, Tolperison $\mathrm{n}=1$ ), meist in Kombination mit weiteren Medikamenten, beteiligt. Die restlichen schweren Vergiftungen wurden durch Hämatologika (Dabigatran $\mathrm{n}=1$, Chloroquin $\mathrm{n}=$ 1 , Eisen $n=1$ ) und Onkologika (Methotrexat $n=2$ ) verursacht.

Bei den vier Todesfällen durch Medikamente waren alle in suizidaler Absicht mit Amlodipin, Propranolol, Escitalopram, Miratazapin, Azathiprin, Opiaten, Phenobarbital, Sertralin in unterschiedlicher Kombination eingenommen worden. Ein weiterer Todesfall trat in Zusammenhang mit Ketamin und Cocain auf, einmal im Rahmen einer suizidalen Handlung mit Schweinfurter Grün, und in einem Fall wurde hochkonzentrierte Essigsäure eingenommen.

\section{L'essentiel en bref}

- En 2017, Tox Info Suisse a répondu à 40310 demandes de renseignements, dont environ 37200 avec exposition et 3100 de nature préventive.

- Plus de 18000 expositions à une substance toxique concernaient des enfants (contre plus de 14000 touchant des adultes), $81 \%$ d'entre eux ayant moins de 5 ans.

- Près des trois quarts (69\%) des intoxications étaient dues aux médicaments, aux produits ménagers ou aux plantes (tab. 1).

- Quatre (57\%) des sept décès enregistrés étaient dus à des intoxications médicamenteuses, les trois autres étant respectivement liés à des produits techniques/professionnels, des drogues et des aliments/boissons (tab. 2).

- Parmi les cas graves, $67 \%$ étaient liés à des médicaments, $15 \%$ à des aliments/boissons et à des drogues et $6 \%$ à des produits ménagers. 
Tabelle 1: Häufigkeit der Vergiftungen beim Menschen nach Noxengruppen (Tox Info Suisse 2017).

\begin{tabular}{|c|c|c|c|c|c|}
\hline $\begin{array}{l}\text { Noxengruppen/ } \\
\text { Altersgruppen }\end{array}$ & Erwachsene & Kinder & $\begin{array}{l}\text { Alter } \\
\text { undefiniert }\end{array}$ & Total & \\
\hline Medikamente & 5861 & 5461 & 18 & 11340 & $34,7 \%$ \\
\hline Haushaltsprodukte & 2735 & 5847 & 24 & 8606 & $26,3 \%$ \\
\hline Pflanzen & 611 & 2149 & 3 & 2763 & $8,4 \%$ \\
\hline $\begin{array}{l}\text { Körperpflegemittel und } \\
\text { Kosmetika }\end{array}$ & 355 & 2008 & 2 & 2365 & $7,2 \%$ \\
\hline $\begin{array}{l}\text { Technische und gewerbliche } \\
\text { Produkte }\end{array}$ & 1691 & 417 & 12 & 2120 & $6,5 \%$ \\
\hline Nahrungsmittel und Getränke & 826 & 693 & 9 & 1528 & $4,7 \%$ \\
\hline $\begin{array}{l}\text { Genussmittel, Drogen und } \\
\text { Alkohol }\end{array}$ & 578 & 487 & 2 & 1067 & $3,3 \%$ \\
\hline $\begin{array}{l}\text { Produkte in Landwirtschaft } \\
\text { und Gartenbau }\end{array}$ & 368 & 355 & 0 & 723 & $2,2 \%$ \\
\hline Pilze & 346 & 243 & 4 & 593 & $1,8 \%$ \\
\hline (Gift-)Tiere & 290 & 120 & 2 & 412 & $1,3 \%$ \\
\hline Tierarzneimittel & 72 & 55 & 0 & 127 & $0,4 \%$ \\
\hline Andere oder unbekannte Noxen & 679 & 382 & 14 & 1075 & $3,3 \%$ \\
\hline Total & 14412 & 18217 & 90 & 32719 & $100 \%$ \\
\hline
\end{tabular}

\section{Genussmittel, Drogen und Alkohol}

Genussmittel, Drogen und Alkohol führten zu einer tödlichen und 37 schweren Vergiftungen. Beim Todesfall kam es in Zusammenhang mit Ketamin und Cocain zu Asystolie mit metabolischer Azidose, mehrmaligen, generalisierten Krampfanfällen und schliesslich Exitus letalis. 12 Fälle betrafen Alkoholvergiftungen, eine Cannabinoide, drei Opioide, neun Halluzinogene und Stimulantien (inkl. Ecstasy) und vier Cocain. Sechs waren die Folge von Gammahydroxybutyrat (GHB), Gammabutyrolacton (GBL) auch in Kombination mit Alkohol oder anderen Drogen. Bei den Alkoholvergiftungen wurden in zwei Drittel der Fälle auch noch Medikamente oder andere Drogen konsumiert. Insgesamt waren über zwei Drittel $(n=25)$ der Patienten männlich. Bei den drei schweren Opiatintoxikationen, zwei davon mit Heroin, stand das typische klinische Bild mit Koma und Atemdepression im Vordergrund, obschon in mindestens zwei Fällen weitere Drogen oder Alkohol mitkonsumiert worden waren.

Bei den neun Patienten, die Halluzinogene (LSD [2]) und Stimulantien (z.B. Amphetamin, Methampheta$\mathrm{min})$, teilweise zusammen mit weiteren Drogen, Medikamenten und/oder Alkohol, konsumiert hatten, traten Agitation/Psychosen, epileptische Anfälle, aber auch Koma auf, dazu Rhabdomyolyse, metabolische Azidose sowie Asystolie.

Von den vier Patienten nach Cocainintoxikation entwickelten drei eine Rhabdomyolyse mit stark erhöhter Creatinkinase. In einem Fall kam es zu stenokardischen Beschwerden mit Myokardinfarkt bzw. Transaminasenerhöhung. Es handelte sich jeweils um Män- ner jünger 36 Jahre. Cocain wurde dabei inhalativ zugeführt.

Von den sechs Patienten nach GHB-Konsum, alle männlich, verloren fünf das Bewusstsein, waren tief komatös und mussten z.T. schutzintubiert werden. Häufig war ein Beikonsum mit anderen Drogen und Alkohol vorhanden. Alle wachten nach wenigen Stunden rasch wieder auf, wie dies nach GHB typischerweise beobachtet wird. Ein Patient entwickelte nach Einnahme von Poppers, die flüchtige Nitrite enthalten, eine schwere Methämoglobinämie mit Werten $>60 \%$.

\section{Pflanzen}

Expositionen mit Pflanzen führten 2017 zu vier schweren Vergiftungen - alle bei Erwachsenen. Ein Mann entwickelte nach Verwechslung von Herbstzeitlosen mit Bärlauch gastrointestinale Symptome mit wässriger Diarrhoe, Hepatopathie und Knochenmarksdepression. Erhöhte Colchizinspiegel bewiesen die Vergiftung mit Colchicum autumnale. Bei schwerer Neutropenie mit Pneumonie musste er intubiert werden und benötigte Vasopressoren. Ein junge Patientin entwickelte einen AV-Block III $^{\circ}$ nach Einnahme von Digitalis purpurea (Roter Fingerhut). Nach repetitiver Kohlegabe traten keine weiteren Komplikationen auf, und es waren dadurch keine Digi-fab-Antikörper notwendig. Eine Patientin mittleren Alters entwickelte nach subkutaner Selbstinjektion von geriebenen Rizinusbohnen vermischt mit Wasser eine lokale Gewebsschädigung mit nekrotisierender Faszitis. Ricin führt durch Inhibition der Proteinsynthese zu Apoptose mit Nekrose. Eine operative Sanierung war notwendig. Bei einem Patienten mittleren Alters kam es nach Verzehr von Khat (Catha edulis) zu einer schweren Psychose, Halluzinationen und Desorientierung.

\section{Haushaltsprodukte}

Bei den Haushaltsprodukten traten 15 schwere Intoxikationen auf, darunter zwei bei Kleinkindern ( $<3$ Jahren). Bei den beiden Kleinkindern kam es nach oraler Einnahme von Backofen- und Grillreiniger zu Epiglottisödem, schweren Verätzungen und Ulzerationen im Ösophagus mit Hospitalisationen länger als eine Woche. Zwei Patienten zeigten nach oraler Aufnahme von javelhaltigen Produkten, welche Natriumhypochlorit enthalten, Verätzungen der Schleimhäute des Gastrointestinaltrakts, entwickelten aber auch eine Aspirationspneumonie. Bei einer erwachsenen $\mathrm{Pa}$ tientin mittleren Alters kam es nach Mischen von Javelwasser mit Essigsäure zu Chlorgasbildung mit Reizwirkung der Atemwege und starkem Sättigungsabfall. 
Tabelle 2: Häufigkeit der Noxengruppen und Vergiftungsschweregrad der auswertbaren ärztlichen Rückmeldungen (Tox Info Suisse 2017) zu Giftkontakt beim Menschen (nur hohe Kausalität), Medikamente nach ATC-Codegruppen.

\begin{tabular}{|c|c|c|c|c|c|c|c|c|c|c|c|c|}
\hline \multirow[b]{2}{*}{ Noxengruppen/Schweregrad } & \multicolumn{5}{|c|}{ Erwachsene } & \multicolumn{5}{|c|}{ Kinder } & \multicolumn{2}{|l|}{ Total } \\
\hline & 0 & $\mathbf{L}$ & M & $\mathbf{S}$ & $\mathrm{T}$ & 0 & $\mathbf{L}$ & M & $\mathbf{S}$ & $\mathrm{T}$ & & \\
\hline Medikamente & 360 & 1060 & 402 & 153 & 4 & 309 & 205 & 46 & 6 & - & 2545 & $60,3 \%$ \\
\hline \multicolumn{13}{|l|}{ davon } \\
\hline Nervensystem & 246 & 873 & 309 & 129 & 2 & 90 & 116 & 31 & 3 & 0 & 1799 & \\
\hline Atemwege & 5 & 38 & 33 & 3 & 0 & 28 & 19 & 2 & 0 & 0 & 128 & \\
\hline Bewegungsapparat & 47 & 63 & 17 & 6 & 0 & 27 & 24 & 4 & 0 & 0 & 188 & \\
\hline Kreislauf & 19 & 26 & 14 & 2 & 2 & 32 & 11 & 1 & 0 & 0 & 107 & \\
\hline Verdauung & 12 & 7 & 11 & 6 & 0 & 44 & 10 & 3 & 0 & 0 & 93 & \\
\hline übrige & 31 & 53 & 18 & 7 & 0 & 88 & 25 & 5 & 3 & 0 & 230 & \\
\hline Haushaltsprodukte & 42 & 159 & 28 & 13 & 0 & 90 & 115 & 11 & 2 & 0 & 460 & $10,9 \%$ \\
\hline Technische und gewerbliche Produkte & 34 & 230 & 40 & 10 & 1 & 11 & 29 & 3 & 2 & 0 & 360 & $8,5 \%$ \\
\hline Genussmittel, Drogen und Alkohol & 15 & 135 & 129 & 35 & 1 & 11 & 14 & 8 & 2 & 0 & 349 & $8,3 \%$ \\
\hline Pflanzen & 11 & 31 & 12 & 4 & 0 & 23 & 15 & 2 & 0 & 0 & 98 & $2,3 \%$ \\
\hline Produkte in Landwirtschaft und Gartenbau & 3 & 10 & 6 & 0 & 0 & 8 & 6 & 0 & 0 & 0 & 33 & $0,8 \%$ \\
\hline Körperpflegemittel und Kosmetika & 8 & 23 & 3 & 1 & 0 & 23 & 34 & 5 & 0 & 0 & 97 & $2,3 \%$ \\
\hline Pilze & 3 & 33 & 32 & 2 & 0 & 10 & 6 & 4 & 0 & 0 & 90 & $2,1 \%$ \\
\hline (Gift-)Tiere & 3 & 18 & 8 & 2 & 0 & 0 & 9 & 2 & 0 & 0 & 42 & $1,0 \%$ \\
\hline Nahrungsmittel und Getränke & 3 & 12 & 10 & 1 & 1 & 7 & 11 & 2 & 0 & 0 & 48 & $1,1 \%$ \\
\hline Tierarzneimittel & 2 & 3 & 3 & 1 & 0 & 1 & 2 & 0 & 0 & 0 & 12 & $0,3 \%$ \\
\hline Andere oder unbekannte Noxen & 3 & 48 & 8 & 5 & 0 & 14 & 7 & 1 & 0 & 0 & 86 & $2,0 \%$ \\
\hline Total & 487 & 1762 & 681 & 227 & 7 & 507 & 453 & 84 & 12 & 0 & 4220 & $100 \%$ \\
\hline
\end{tabular}

Schweregrad des Verlaufs: $\mathrm{O}=$ asymptomatisch, $\mathrm{L}=$ leicht, $\mathrm{M}=$ mittel, $\mathrm{S}=$ schwer, $\mathrm{T}=$ tödlich.

Sechs Patienten entwickelten nach oraler Einnahme von Ethylenglykol eine schwere Azidose. Einige wurden komatös und mussten intubiert werden. In allen Fällen wurde als Antidot Fomepizol oder Ethanol verabreicht. Auch war bei allen Patienten ein Nierenersatzverfahren zur Elimination von Ethylenglykol und Korrektur der schweren Azidose mittels Hämodialyse notwendig.

\section{Kosmetika und Körperpflegemittel}

Durch Kosmetika und Körperpflegemittel waren 2017 eine schwere und acht mittelschwere Vergiftungen (fünf davon bei Kindern) zu verzeichnen. Bei einer erwachsenen Patientin kam es nach Einnahme von Nagellackentferner mit Isopropanol zu Koma, Aspirationspneumonie und schwerer Rhabdomyolyse mit akuter Niereninsuffizienz nach Bewusstlosigkeit und Liegetrauma. Bei fünf Kindern kam es nach akzidenteller okulärer Applikation von Shampoos zu starker Augenreizung.

\section{Nahrungsmittel und Getränke}

Mit Nahrungsmitteln und Getränken kam es $2017 \mathrm{zu}$ einem tödlichen, einem schweren und zwölf mittelschweren Vergiftungen. Ein Patient wies nach Essigsäureeinnahme schwere gastrointestinale Verätzungen mit nachfolgendem Multiorganversagen auf, woran er in weiterer Folge verstarb. Eine adoleszente Patientin entwickelte nach beabsichtigtem Gebrauch von Muskatnuss eine schwere Agitation (durch die mescalinähnliche Substanz Myristicin) in Kombination mit Tachykardie, Mundtrockenheit, Miosis und Tachypnoe. Die mittelschweren Intoxikationen waren durch Meeresfrüchte, Muskatnuss (als Pulver und in Gebäck [3]), Chilischoten mit Capsaicin, konzentrierten Essig und koffeinhaltige Nahrungsergänzungsmittel allesamt nach oraler Einnahme bedingt. Dabei standen gastrointestinale Symptome neben ZNS-Symptomen im Vordergrund. Bei zwei Kleinkindern kam es nach nitrathältigem Gemüsebrei zu einer Methämoglobinämie $>30 \%$, welche sich nach Verabreichung von Methylenblau 1-2 mg/kg Körpergewischt besserte. Nitrate, die in Gemüse wie v.a. Fenchel, Karotten, Kohlrabi, Spinat und Mangold enthalten sind, können durch Bakterien in Nitrite umgewandelt werden, welche Methämoglobinbildner sind [4].

\section{Technisch-gewerbliche Produkte}

$\mathrm{Zu} 12$ schweren und einer tödlichen Vergiftung kam es durch technisch-gewerbliche Produkte, mit zwei Ausnahmen alle bei Erwachsenen. Der Todesfall war die Folge einer Einnahme von Schweinfurter Grün, einer trivalenten Arsenverbindung, mit schwerem Schock und schwerer metabolischer Azidose. Bei den beiden Kinderfällen handelte es sich um orale Vergiftungen, 
beide mit basenhaltigen Produkten (Natronlauge bzw. Melkmaschinenreiniger) und daraus resultierenden lokalen Laugenverätzungen im Ösophagus. Von den zehn schweren Vergiftungen bei Erwachsenen waren vier durch Ethylenglykol nach oraler Aufnahme bedingt. Alle Patienten entwickelten eine schwere metabolische Azidose mit Koma oder Niereninsuffizienz und erhielten als Antidot Fomepizol oder Ethanol und eine Nierenersatztherapie. Die restlichen Fälle waren durch Benzin, Aceton, Ammoniakwasser, Natriumhydroxid, Zement (okuläre Exposition) verursacht. In 40\% war die Exposition beruflich.

\section{Stoffe in Landwirtschaft und Gartenbau}

Mit Stoffen in Landwirtschaft und Gartenbau ereigneten sich keine schweren Vergiftungen oder Todesfälle. Es wurden im Jahr 2017 sechs mittelschwere Fälle allesamt bei Erwachsenen verzeichnet. Bei einer weiblichen Patientin kam es zu einer Jauchgasexposition mit nachfolgender Schwefelwasserstoffvergiftung (H2S) und Synkope. Durch das Reizgas werden die Zellatmung und die Cytochromoxydase blockiert, und es kommt $\mathrm{zu}$ einer raschen Bewusstlosigkeit. Eine Patientin erlitt Blutungskomplikationen nach Einnahme von Difenacoum, einem Rodentizid aus der Gruppe der Vitamin-K-Antagonisten.

\section{Giftige Tiere}

Insgesamt kam es 2017 in zehn Fällen zu mittelschweren und zweimal zu schweren Symptomen nach Bissen oder Stichen durch giftige Tiere (7 Schlangen [5], 1 Fisch, 1Spinne, 2 Insekten und 1 Qualle); zwei der mittelschweren traten bei Kindern auf. Die schweren Fälle - beide bei Erwachsenen - waren durch Bisse von einheimischen Vipern, einmal von einer Aspisviper und einmal von einer Kreuzotter, verursacht. Der eine Vipernbiss (Hand) führte zu einer Rötung und Schwellung im Bereich der Bissstelle und wurde durch ein Angioödem verkompliziert, worauf die Antidotgabe erfolgte. Beim anderen Vipernbiss (Hand) kam es zu einer Ausbreitung der Schwellung. Auch hier wurde das spezifische Antivenin verabreicht. Bei den fünf mittelschweren Giftschlangenbissen waren alle durch einheimische Vipern (Vipera aspis oder V. berus) in der freien Natur verursacht, alle Bisse erfolgten ebenfalls in die Hand - vorwiegend war der Zeigefinger betroffen. Bei allen kam es zu ausgeprägten Lokalsymptomen (Schmerzen, Schwellung), z.T. mit systemischen Zeichen (Nausea, Erbrechen, Schwindel). Zwei davon erhielten das Antivenin, und bei allen war der weitere Verlauf günstig. Die Hospitalisationszeiten betrugen zwischen einem und drei Tagen.

\section{Pilze}

2017 ereigneten sich zwei schwere Pilzvergiftungen bei Erwachsenen. In einem Fall ass ein betagter Mann nicht identifizierte Pilze und entwickelte Schwindel, Nausea und Emesis. In der weiteren Folge kam es zu Symptomen eines Pantherina-Syndroms. Dieses wird durch Amanita muscaria (Fliegenpilz), Amanita pantherina (Pantherpilz), Amanita gemmata (Narzissengelber Wulstling), Amanita regalis (Königsfliegenpilz) verursacht. Es äussert sich vor allem in gastrointestinalen Symptomen sowie Halluzinationen, Erregunszuständen, aber auch tiefem Koma mit peripheren anticholinergen Symptomen (Mydriase, Tachykardie, Mundtrockenheit, warm-trockene Haut). Im zweiten Fall ass eine ebenfalls betagte Frau amatoxinhaltige Pilze. Bei klinischer Emesis und blutiger Diarrhoe mit Bauchschmerzen sowie laborchemisch stark erhöhten Transaminasen wurden Silibinin und $\mathrm{N}$-Acetylcystein verabreicht. Die Patientin erholte sich wieder, und es war keine Listung für eine Lebertransplantation notwendig. Es handelte sich in beiden Fällen um selbst gesammelte und nicht kontrollierte Pilze.

\section{Andere Noxen}

In einem Fall kam es zu versehentlicher Einnahme von Diethylenglykol mit Multiorganversagen mit akuter Niereninsuffizienz und neurologischen Symptomen. Eine andere erwachsene Patientin erlitt im Rahmen einer Wasserintoxikation mit sieben Litern eine schwere Hyponatriämie mit rezidivierenden generalisierten epileptischen Anfällen. Eine junge Patientin wurde nach vermutlicher KO-Tropfen-Exposition rasch tief komatös (GCS 4).

Weiterführende Angaben

1 Der Schweregradbewertung durch Tox Info Suisse liegt der Poisoning Severity Score zugrunde (Persson HE, et al. Clin Toxicol. 1998; 36:205-13). Er beruht auf der Bewertung von Einzelsymptomen und -befunden nach festen Kriterien. Eine Vergiftung ist demnach leicht, wenn Symptome auftreten, die wenig beeinträchtigend sind und in der Regel spontan wieder verschwinden. Eine mittelschwere Intoxikation liegt dann vor, wenn behandlungswürdige und länger anhaltende Symptome vorhanden sind. Schwere Symptome sind ausnahmslos immer behandlungswürdig, lebensbedrohlich und/oder führen zu bleibenden Beeinträchtigungen

2 LSD = Lysergsäurediethylamid.

3 Die «Nervenkekse» nach Hildegard von Bingen enthielten $45 \mathrm{~g}$ Muskat pro Kilogramm Mehl (Beck TA, Marty H. Swiss Med Forum. 2001;1:1287-8).

4 Hofer KE, Faber K, Rauber-Lüthy C. Methemoglobinemia in children reported to Tox Info Suisse: a retrospective case series [abstract]. Swiss Med Wkly. 2017;147(Suppl 222):41.

5 Die Zahlen zu den Schlangenbissen 2017 finden sich bei www.antidota.ch 